\title{
Diabetes health management strategy based on internet plus graded diagnosis and treatment strategy
}

\author{
Guiwen Liang $^{1 \#}$, Haiyan Jiang ${ }^{1 \#}$, Chunxia Huang ${ }^{1}$, Xianfeng Que ${ }^{1}$, Jianzhong Tang ${ }^{1,2}$, Juying Lu ${ }^{1}$, \\ Jianlin Gao ${ }^{1}$
}

${ }^{1}$ Center for Health and Disease Management, Affiliated Hospital of Nantong University, Nantong, China; ${ }^{2}$ Department of General Practice, Affiliated Hospital of Nantong University, Nantong, China

Contributions: (I) Conception and design: J Gao, J Lu; (II) Administrative support: H Jiang; (III) Provision of study materials or patients: J Tang, X Que; (IV) Collection and assembly of data: G Liang; (V) Data analysis and interpretation: G Liang, C Huang; (VI) Manuscript writing: All authors; (VII) Final approval of manuscript: All authors.

"These authors contributed equally to this work.

Correspondence to: Prof. Juying Lu; Prof. Jianlin Gao. Center for Health and Disease Management, Affiliated Hospital of Nantong University, 20 West Temple Road, Nantong, China. Email: tdfylujy@163.com; tdfygaoj1@163.com.

\begin{abstract}
Background: Type 2 diabetes (T2D) is a widespread chronic disease with high rates of morbidity and mortality worldwide. Managing risk factors can effectively prevent acute and chronic complications, improve quality of life, and reduce mortality. Therefore, implementation of a diabetes health management strategy is urgently needed. Emerging medical technologies have strengthened communication between patients and clinicians. The establishment and improvement of the graded diagnosis and treatment system has promoted the prevention, treatment, and management of diabetes.
\end{abstract}

Methods: A total of 300 patients diagnosed with T2D in the Health Management Center at the Affiliated Hospital of Nantong University were randomly divided into two groups: an internet plus graded diagnosis and treatment strategy group, and a control group. After 6 months, the physiological parameters, management indices, and complications were compared between the groups.

Results: Physiological indicators, such as body mass index (BMI), waist circumference, triglycerides, lowdensity lipoprotein, systolic and diastolic blood pressure, and blood glucose were significantly alleviated in the Internet plus graded diagnosis and treatment strategy group. Management indicators (such as blood glucose monitoring compliance rate, diet control compliance rate, and exercise compliance rate) also improved substantially. The incidence of hypoglycemia was notably increased compared to the control group.

Conclusions: The new health management strategy for diabetes can improve lifestyle, ameliorate physiological indicators, reduce the complication rate, and form a virtuous cycle. This provides a positive impact on the entire life-cycle health management of diabetes, and is worthy of further promotion.

Keywords: Internet; graded diagnosis and treatment; diabetes; health management strategy

Submitted Aug 20, 2020. Accepted for publication Nov 06, 2020.

doi: 10.21037/apm-20-1996

View this article at: http://dx.doi.org/10.21037/apm-20-1996

\section{Introduction}

Diabetes is one of the most prevalent chronic diseases in the world. It has high rates of mortality and morbidity that greatly impacts the health of patients globally (1).
The occurrence and development of type 2 diabetes (T2D) is accompanied by other micro- and macrovascular diseases (2). Hyperlipidemia and hypertension are common comorbidities in patients with $\mathrm{T} 2 \mathrm{D}$, and the interaction of comorbidities significantly increases the 
risks to patients (3). Previous studies have shown that the continuous and stable control of blood glucose (4), blood pressure (5) and blood lipids (6) can significantly reduce the occurrence and development of acute and chronic diabetes complications. The most recent American Diabetes Association (ADA) guidelines suggest that glycosylated hemoglobin (HbA1c) should be controlled below 7.0\%, and blood pressure should be controlled below 140/ $90 \mathrm{mmHg}$ (blood pressure of patients with cardiovascular risk factors should be controlled below 130/90 $\mathrm{mmHg}$ ) (7). Low density lipoprotein cholesterol (LDL-C) control at $100 \mathrm{mg} / \mathrm{dL}$ is necessary for most adult diabetes patients and can significantly improve quality of life.

It is also crucial to enhance the management of patients with T2D. In the process of long-term health management, clinicians and other medical staff should aim to constantly broaden their knowledge and develop new technologies and methods (8). It is particularly prudent to augment the education of patients, strengthen doctor-patient interaction, implement dynamic changes, and streamline the entire patient management process. Diabetic patients should also aim to cooperate with medical staff and improve their selfmanagement ability during the treatment process (9).

The progress of science and technology promotes the advancement of medical treatment and health management. With the continuous development of information communication, artificial intelligence, and other technologies, newly developed technologies in chronic disease health management offer numerous advantages over traditional management strategies, including remote communication, continuous monitoring, wide coverage, and self-support (10-12). However, there is still a lack of authoritative guidance and application planning regarding the mechanisms of combining diabetes health management with information and communication technology (13).

In recent years, owing to the rapid developments in cellular and mobile technology, the application value of mobile phone technology has accelerated. In 2015, China put forward the strategy of "Internet plus", which was a powerful boost to the development of mobile medical technology. The establishment of hierarchical diagnosis and treatment systems is an important measure to reasonably allocate medical resources and balance basic medical and health services at this stage, and is crucial for promoting the development of medical and health undertakings in the long-term. China's provinces and cities should adjust measures to local conditions, promote the work of hierarchical diagnosis and treatment, establish an ideal hierarchical diagnosis and treatment policy system, and construct a hierarchical diagnosis and treatment system in line with Chinese national conditions. The pilot areas focused on chronic diseases and carried out graded diagnosis and treatment of hypertension and T2D.

Under the guidance of technological innovation and policy support towards development, we innovatively adopted the Internet plus hierarchical diagnosis and treatment strategy to manage the health of diabetes patients, and integrated the convenience and practicability of high-speed, real-time, and hierarchical treatment in the Internet plus framework. Based on the structure of our hospital's health management center, as well as the coordinate location of our hospital and the United Medical Association Hospital, specialists perform health education, hierarchical diagnosis and treatment, remote consultation, and provide long-term precise management of diabetes using Internet mobile terminal technology. We present the following article in accordance with the CONSORT reporting checklist (available at http://dx.doi.org/10.21037/ apm-20-1996).

\section{Methods}

This study has been approved by the Ethics Committee of the Affiliated Hospital of Nantong University (Approval number: 2019-L002), and all participants and their families signed the informed consent documents. The study was conducted in accordance with the Declaration of Helsinki (as revised in 2013).

\section{Patient selection}

From January 2018 to March 2019, 300 patients with T2D who were confirmed by physical examination in the health management center of Affiliated Hospital of Nantong University were selected.

\section{Inclusion criteria}

(I) Accord with the diagnostic and treatment standards for diabetes promulgated and updated by the American Diabetes Association (ADA) in 2017 (14): fasting blood glucose $(\mathrm{FPG}) \geq 7.0 \mathrm{mmol} / \mathrm{L}$; or 2 -hour blood glucose $\geq 11.1 \mathrm{mmol} / \mathrm{L}$ by oral glucose tolerance test (OGTT); or HbA1c $\geq 6.5 \%$ [National Glycohemoglobin Standardization Program (NGSP) certified and standardized measurement according to the Diabetes Control and Complications Trial 
(DCCT) test]; or diabetes symptoms + plasma glucose level (at any time) $\geq 11.1 \mathrm{mmol} / \mathrm{L}$. Note: if there were no symptoms of diabetes, confirmation was required twice. (II) Voluntary participation in the study and signed informed consent. (III) Age between 20 and 70 years. (IV) Ability to use smartphones.

\section{Exclusion criteria}

(I) Patients who were unwilling to cooperate. (II) Patients with poor treatment compliance. (III) Patients with acute complications of diabetes mellitus or other serious systemic diseases.

\section{Shedding criteria}

(I) Patients who quit voluntarily. (II) Severe acute diabetic complications or other serious systemic diseases occurring during the study period.

\section{General patient information and measurement parameters}

The general patient data included name, gender, age, occupation, education level, etc. The measurement parameters included height, weight, waist circumference, blood pressure (systolic and diastolic), fasting plasma glucose (FPG), 2-hour postprandial plasma glucose $(2 \mathrm{~h}$ PG), HbA1c, total cholesterol (TC), total triglycerides (TG), low-density lipoprotein (LDL-C), and high-density lipoprotein (HDL-C). Patient information was entered into the management platform.

\section{Groups and interventions}

A total of 300 patients with T2D were randomly divided into an internet plus graded diagnosis and treatment strategy group (internet + group) and a control group, with 150 patients in each group. The data of the two groups were obtained from the health management center at the Affiliated Hospital of Nantong University.

The Internet plus classification treatment group was managed using Internet mobile terminal technology. Firstly, point-to-point and point-to-face connection between doctors and patients was established via the WeChat platform. Patients could also nominate any specialist in the Medical Association. Furthermore, specialists could set individual and precise physiological parameters to calibrate treatment goals for patients using the mobile point-to- point terminal. Based on these treatment goals, doctors were able to set different diet, exercise, and medication packages. If, after taking various measurements, the patient's physiological parameters were not seen to have significantly improved, or if complications occurred, the medical alliance specialist referred patients to the specialist experts at our hospital for remote consultation or outpatient diagnosis and treatment. At the same time, treatment enhancement opinions will be sent to the original management doctors of the Medical Union in real time through the mobile terminal, thereby providing a hierarchical diagnosis and treatment interconnection. Moreover, we encouraged the use of the WeChat application on the mobile network, and the establishment of groups according to age, disease condition, and treatment plan. Health management doctors sent health education, blood glucose monitoring, drug treatment, and other information to individuals or groups, so as to improve the self-management ability of diabetes patients. Diabetic patients could also share their experiences and build confidence using group chat features.

The control group adopted the traditional management mode. The patients independently recorded blood glucose levels and attended outpatient or emergency departments regularly, or in cases of unstable blood glucose or other irregularities. After 6 months, the physiological indices were measured, the incidence of complications was calculated, and the management parameters (including blood glucose monitoring, diet control and exercise execution) were recorded. Statistical analysis was performed.

\section{Indicators included}

The blood glucose index (FPG, 2 h PG, and HbA1c), body mass index (BMI), waist circumference, blood pressure, and blood lipids were measured at baseline and following the 6-month intervention in both groups. At the conclusion of the intervention, the management parameters (including the compliance rates of blood glucose monitoring, diet control, and exercise) and hypoglycemia incidence rate were calculated in both groups.

\section{Statistical analysis}

GraphPad 5.0 and IBM SPSS 20 statistical software were used for data analysis. The measurement data were expressed as mean \pm standard deviation, and the comparison between the groups was performed by $t$-test. The count data was analyzed by Chi-square test. $\mathrm{P}<0.05$ indicated that 
Table 1 Baseline data analysis of internet plus grading treatment group and control group (mean \pm SD)

\begin{tabular}{|c|c|c|c|c|}
\hline Index & Internet + group $(n=150)$ & Control group $(n=150)$ & Statistics & $P$ value \\
\hline Gender (male/female) & $94 / 56$ & $102 / 48$ & $\chi^{2}=0.942$ & 0.332 \\
\hline $\mathrm{BMI}\left(\mathrm{kg} / \mathrm{m}^{2}\right)$ & $27.37 \pm 3.42$ & $27.82 \pm 4.16$ & $t=0.555$ & $>0.999$ \\
\hline Waist (cm) & $102.33 \pm 15.23$ & $104.67 \pm 14.52$ & $t=0.251$ & 0.283 \\
\hline Diastolic blood pressure $(\mathrm{mmHg})$ & $75.87 \pm 17.42$ & $78.24 \pm 16.68$ & $t=0.413$ & 0.266 \\
\hline $\mathrm{TC}(\mathrm{mmol} / \mathrm{L})$ & $6.54 \pm 1.13$ & $6.47 \pm 1.31$ & $t=0.144$ & $>0.999$ \\
\hline $\mathrm{TG}(\mathrm{mmol} / \mathrm{L})$ & $2.83 \pm 0.92$ & $2,93 \pm 1.12$ & $t=0.117$ & $>0.999$ \\
\hline LDL-C (mmol/L) & $4.13 \pm 0.42$ & $4.27 \pm 0.31$ & $\mathrm{t}=0.717$ & $>0.999$ \\
\hline 2 h PG (mmol/L) & $12.22 \pm 1.87$ & $12.32 \pm 1.93$ & $t=0.161$ & $>0.999$ \\
\hline HbA1c (\%) & $9.03 \pm 1.55$ & $9.16 \pm 1.42$ & $t=0.449$ & $>0.999$ \\
\hline
\end{tabular}

BMI, body mass index; TC, total cholesterol; TG, total triglycerides; LDL-C, low-density lipoprotein; HDL-C, high-density lipoprotein; FPG, fasting plasma glucose; 2 h PG, 2-hour postprandial plasma glucose.

the difference was statistically significant.

\section{Results}

\section{Baseline data analysis}

There were no significant differences in gender, age, BMI, waist circumference, blood pressure, blood lipids, and blood sugar (FPG, 2 h PG, and HbAlc) between the two groups (Table 1).

\section{Analysis of physiological parameters after 6 months}

In the Internet plus grading treatment group, three cases voluntarily withdrew, and 16 cases were lost following the 6-month intervention. Compared with the control group, the BMI, waist circumference, blood lipids, triglycerides, LDL, systolic blood pressure, and blood sugar decreased markedly in the treatment group. However, there were no significant differences in total cholesterol, HDL, diastolic blood pressure, and $\mathrm{HbA1c}$ between the two groups (Table 2).

\section{Analysis of the management indicators}

After 6 months, the management indicators (including blood glucose monitoring compliance rate, dietary control compliance rate, and exercise compliance rate) and hypoglycemia incidence rate results showed that the compliance rates of blood sugar monitoring, diet, and exercise in the Internet plus grading treatment group were notably better than the control group. The incidence of hypoglycemia was also substantially higher than the control group, and the difference was statistically significant (Table 3).

\section{Discussion}

Diabetes is one of the most prevalent chronic diseases threatening human health worldwide. It exhibits characteristics such as being indistinguishable and preventing difficulties, and patients with diabetes experience a high cost of treatment, lifelong use of medication, and serious complications. Moreover, its high rates of incidence, disability, and mortality are critical public health issues worthy of global attention (15). It is particularly important to conduct health management strategy research on diabetes, and especially T2D (16). With the rapid progress of Internet and microelectronics technology, the Internet plus-based remote health management system for mobile terminals has gradually been established, and has been particularly important for the management of chronic 
Table 2 Comparison of physiological parameters between the two groups after 6 months (mean \pm SD)

\begin{tabular}{|c|c|c|c|c|}
\hline Index & Internet + group $(n=131)$ & Control group $(n=121)$ & Statistics & $P$ value \\
\hline Waist (cm) & $93.03 \pm 7.61$ & $101.04 \pm 9.07$ & $t=7.614$ & $<0.001$ \\
\hline Systolic blood pressure (mmHg) & $125.25 \pm 8.63$ & $135.46 \pm 14.67$ & $t=6.798$ & $<0.001$ \\
\hline Diastolic blood pressure $(\mathrm{mmHg})$ & $66.43 \pm 6.44$ & $66.76 \pm 6.58$ & $t=0.403$ & 0.687 \\
\hline TG (mmol/L) & $1.21 \pm 0.74$ & $2.11 \pm 1.18$ & $t=7.273$ & $<0.001$ \\
\hline LDL-C (mmol/L) & $2.31 \pm 0.72$ & $3.30 \pm 1.02$ & $t=8.912$ & $<0.001$ \\
\hline HDL-C (mmol/L) & $0.93 \pm 0.36$ & $0.95 \pm 0.34$ & $\mathrm{t}=-0.582$ & 0.561 \\
\hline $\mathrm{FPG}(\mathrm{mmol} / \mathrm{L})$ & $6.10 \pm 1.13$ & $7.84 \pm 1.07$ & $\mathrm{t}=12.548$ & $<0.001$ \\
\hline
\end{tabular}

BMI, body mass index; TC, total cholesterol; TG, total triglycerides; LDL-C, low-density lipoprotein; HDL-C, high-density lipoprotein; FPG, fasting plasma glucose; 2 h PG, 2-hour postprandial plasma glucose.

Table 3 Comparison of management indicators and complication rate after 6 months

\begin{tabular}{|c|c|c|c|c|}
\hline Index & Internet + group $(n=131)$ & Control group $(n=121)$ & Statistics & $P$ value \\
\hline Diet control compliance rate & $97 / 131$ & $55 / 121$ & $\chi^{2}=21.482$ & $<0.001$ \\
\hline Exercise compliance rate & $85 / 131$ & $53 / 121$ & $\chi^{2}=11.287$ & 0.001 \\
\hline Incidence of hypoglycemia & $39 / 131$ & $22 / 121$ & $\chi^{2}=4.605$ & 0.032 \\
\hline
\end{tabular}

diseases such as T2D and hypertension (17). Technological advancements improve the health management of diabetic patients in medical institutions, and help to resolve problems such as shortage of chronic disease management personnel and insufficient management in medical institutions. It also allows diabetic patients to enhance their self-management ability, especially with regards to diet, exercise, and other aspects closely related to the improvement of physiological parameters (18).

Specifically, this technology allows patients with poor blood glucose control or complications to express their consultation needs to the community health service center or directly ask the tertiary hospital specialist for remote diagnosis and treatment. This improves the idle primary medical and health resources, alleviates problems such as shortages of medical resources and experts in tertiary hospitals, and ensures the continuous implementation of hierarchical diagnosis and treatment policy.
In our study, we innovatively adopted the Internet plus grading health management strategy to conduct a 6-month intervention for patients with T2D. The control group was managed according to the traditional diabetes management approach. The results showed that the BMI, waist circumference, blood lipids, triglycerides, LDL, systolic and diastolic blood pressure, and blood sugar decreased significantly in the treatment group. After 6 months of management, most of the physiological parameters and management indices of the Internet plus grading treatment group were better than those of the control group. Systolic blood pressure and pulse pressure were significantly lower in the treatment group compared with the control group. The underlying cause of this was effective blood pressure control and rapid recovery. Also, FPG and 2h PG decreased markedly in the treatment group compared with the control group, yet some patients did not reach an ideal state, indicating that the intervention time was insufficient. There 
was no significant difference in $\mathrm{HbAlc}$ between the two groups. We considered that since HbAlc reflects the blood glucose level over the preceding months, the short study duration meant that differences in HbAlc levels between the groups could not be observed. Compared with the control group, TC and TG were substantially decreased in the treatment group. However, some patients in the treatment group still exhibited higher than the normal values of TC and TG, which we also attributed the short duration of intervention.

It is worth noting that the incidence of hypoglycemia in the Internet plus classification treatment group was higher than that in the control group. We speculate that the patients in the treatment group had higher rates of dietary control and exercise compliance, as well as greater awareness of serious diabetes complications and tighter controls on their blood sugar levels. The lack of effective education, timely formation of a healthy lifestyle, and correct blood glucose management in the control group resulted in a lower incidence of hypoglycemia and greater incidence of hyperglycemia. Therefore, it is necessary to further extend the intervention time, continue to conduct research, and pay greater attention to the high incidence of hypoglycemia complications.

The new internet plus and mobile terminal technology has changed the concept of healthcare for diabetic patients, allowed for timely answers to their questions and doubts, corrected bad living habits in real time, instituted positive regular lifestyle habits (including blood glucose monitoring, healthy diet, and moderate exercise), and promoted improvement in various physiological parameters. The hierarchical diagnosis and treatment based on the Internet plus technology further strengthens the health management of diabetic patients, facilitates their contact and interaction with doctors at all levels, reduces the cost of diagnosis and treatment, and improves the medical experience of patients.

It is important to recognize that $\mathrm{T} 2 \mathrm{D}$ is a prevalent chronic disease that requires lifelong monitoring and treatment, and has a substantial time, personnel, and economic burden. In this study, we responded to the national call as a three-grade public hospital, and actively participated in the establishment of a consolidated medical body, promoted quality medical resources to provide to community hospitals, and helped to initiate a reasonable and orderly diabetes classification and treatment mode. Health management can greatly reduce the burden of T2D, and effectively improve physiological indicators, significantly reduce complications, and produce good economic and social benefits. In future, we aim develop a fully functional mobile terminal application with the ability to provide the following: analyze various physiological parameters and blood glucose monitoring data, provide early screening for diabetes cases, set diet control and exercise/fitness plans, rapidly classify diagnosis and treatment, adjust treatment plans, quickly detect diabetes complications and provide early warning, promote communication and supervision among patients with diabetes, and improve patient compliance to diagnosis and treatment plans. We believe this could realize the ideal holistic and individualized management of diabetes.

However, there are some limitations in this study that should be noted. Firstly, the intervention time was only 6 months, which is a relatively short duration for chronic disease management. Secondly, the number of patients receiving the new health management strategy was 150 . This was a relatively small sample size, especially considering the additional voluntary patient exits and losses. Also, this study mainly relied on the mobile WeChat application for point-to-point and point-toface communication and interaction between doctors and patients. At present, we lack the corresponding exclusive application for real-time accurate management. Finally, we still need to take into account some physiological and management indicators, including a variety of hypoglycemia complications, so that our analysis and research is more comprehensive.

\section{Conclusions}

The results of our study demonstrate that a new Internet plus grading health management strategy can provide accurate and individualized management of patients with T2D. The physiological and management indicators of patients improved significantly, and the incidence of complications was markedly reduced. Therefore, the new treatment and health management strategy is worthy of further promotion and clinical application.

\section{Acknowledgments}

Funding: This study was supported by the Nantong Science and Technology Project (MS12019033, MS22019022), the Nantong basic health research project (2020JCC032), and the Nantong Clinical Medicine Research Center (HS2018004, HS2019005). 


\section{Footnote}

Reporting Checklist: The authors have completed the CONSORT reporting checklist. Available at http://dx.doi. org/10.21037/apm-20-1996

Data Sharing Statement: Available at http://dx.doi. org/10.21037/apm-20-1996

Conflicts of Interest: All authors have completed the ICMJE uniform disclosure form (available at http://dx.doi. org/10.21037/apm-20-1996). The authors have no conflicts of interest to declare.

Ethical Statement: The authors are accountable for all aspects of the work in ensuring that questions related to the accuracy or integrity of any part of the work are appropriately investigated and resolved. This study has been approved by the Ethics Committee of the Affiliated Hospital of Nantong University (Approval number: 2019L002), and all participants and their families signed the informed consent documents. The study was conducted in accordance with the Declaration of Helsinki (as revised in 2013).

Open Access Statement: This is an Open Access article distributed in accordance with the Creative Commons Attribution-NonCommercial-NoDerivs 4.0 International License (CC BY-NC-ND 4.0), which permits the noncommercial replication and distribution of the article with the strict proviso that no changes or edits are made and the original work is properly cited (including links to both the formal publication through the relevant DOI and the license). See: https://creativecommons.org/licenses/by-nc-nd/4.0/.

\section{References}

1. International Hypoglycaemia Study Group. Hypoglycaemia, cardiovascular disease, and mortality in diabetes: epidemiology, pathogenesis, and management. Lancet Diabetes Endocrinol 2019;7:385-96.

2. Henning RJ. Type-2 diabetes mellitus and cardiovascular disease. Future Cardiol 2018;14:491-509.

3. Low Wang CC, Hess CN, Hiatt WR, et al. Clinical Update: Cardiovascular Disease in Diabetes Mellitus: Atherosclerotic Cardiovascular Disease and Heart Failure in Type 2 Diabetes Mellitus - Mechanisms, Management, and Clinical Considerations. Circulation
2016;133:2459-502.

4. Giugliano D, Maiorino MI, Bellastella G, et al. Type 2 diabetes and cardiovascular prevention: the dogmas disputed. Endocrine 2018;60:224-8.

5. Vargas-Uricoechea H, Cáceres-Acosta MF. Blood pressure control and impact on cardiovascular events in patients with type 2 diabetes mellitus: A critical analysis of the literature. Metas de control de la presión arterial e impacto sobre desenlaces cardiovasculares en pacientes con diabetes mellitus tipo 2: un análisis crítico de la literatura. Clin Investig Arterioscler 2019;31:31-47.

6. Prídavková D, Samoš M, Bolek T, et al. Type 2 Diabetes, Atrial Fibrillation, and Direct Oral Anticoagulation. J Diabetes Res 2019;2019:5158308.

7. American Diabetes Association. Pharmacologic Approaches to Glycemic Treatment: Standards of Medical Care in Diabetes. Diabetes Care 2020;43:S98-110.

8. Wang DD, Hu FB. Precision nutrition for prevention and management of type 2 diabetes. Lancet Diabetes Endocrinol 2018;6:416-26.

9. Chatterjee S, Davies MJ, Heller S, et al. Diabetes structured self-management education programmes: a narrative review and current innovations. Lancet Diabetes Endocrinol 2018;6:130-42.

10. Jia W, Weng J, Zhu D, et al. Standards of medical care for type 2 diabetes in China 2019. Diabetes Metab Res Rev 2019;35:e3158.

11. Rinaldi G, Hijazi A, Haghparast-Bidgoli H. Cost and costeffectiveness of mHealth interventions for the prevention and control of type 2 diabetes mellitus: A systematic review. Diabetes Res Clin Pract 2020;162:108084.

12. Moonian O, Jodheea-Jutton A, Khedo KK, et al. Recent advances in computational tools and resources for the selfmanagement of type 2 diabetes. Inform Health Soc Care 2020;45:77-95.

13. Abhari S, Niakan Kalhori SR, Ebrahimi M, et al. Artificial Intelligence Applications in Type 2 Diabetes Mellitus Care: Focus on Machine Learning Methods. Healthc Inform Res 2019;25:248-61.

14. American Diabetes Association 2. Classification and Diagnosis of Diabetes. Diabetes Care 2017;40:S11-24.

15. Uusitupa M, Khan TA, Viguiliouk E, et al. Prevention of Type 2 Diabetes by Lifestyle Changes: A Systematic Review and Meta-Analysis. Nutrients 2019;11:2611.

16. Taylor R, Al-Mrabeh A, Sattar N. Understanding the mechanisms of reversal of type 2 diabetes. Lancet Diabetes Endocrinol 2019;7:726-36.

17. Jeffrey B, Bagala M, Creighton A, et al. Mobile phone 
applications and their use in the self-management of Type 2 Diabetes Mellitus: a qualitative study among app users and non-app users. Diabetol Metab Syndr 2019;11:84.

18. Chester B, Babu JR, Greene MW, et al. The effects of popular diets on type 2 diabetes management. Diabetes Metab Res Rev 2019;35:e3188.

(English Language Editor: A. Kassem)

Cite this article as: Liang G, Jiang H, Huang C, Que X, Tang J, Lu J, Gao J. Diabetes health management strategy based on internet plus graded diagnosis and treatment strategy. Ann Palliat Med 2020;9(6):3915-3922. doi: 10.21037/apm-20-1996 